COMUNICACIÓN BREVE

\title{
Características epidemiológicas y clínicas de pacientes internados con COVID-19 en la Ciudad de Buenos Aires
}

\author{
Recibido: 2/12/20 Aceptado: 17/12/20
}

Gabriela Poblete ${ }^{1}$, William Cox ${ }^{1}$, Alberto Dasso ${ }^{2}$, Sandra Begher ${ }^{1}$.

\section{RESUMEN}

Introducción: La pandemia de COVID-19 representa un desafío para la salud mundial y continúa en investigación.

Objetivo: Describir las características epidemiológicas, demográficas, clínicas y la mortalidad por todas las causas de pacientes internados con COVID-19 en un establecimiento de salud privado de la Ciudad de Buenos Aires.

Materiales y métodos: Se realizó un estudio retrospectivo, de corte transversal y descriptivo entre el 3 de marzo y el 8 de julio de 2020. Se incluyeron pacientes adultos con diagnóstico confirmado de COVID-19 por RT-PCR de hisopado nasofaríngeo internados en sala general y unidad de terapia intensiva (UTI) del Sanatorio San José. Se analizaron las características epidemiológicas, demográficas (edad, sexo, ocupación, procedencia, residencia), clínicas y mortalidad por todas las causas. Las variables continuas fueron descriptas con mediana y rango intercuartilo (RIC) y las variables categóricas con número y porcentaje. Se utilizó el programa STATA v 13.0 .

Resultados: Se incluyeron 118 pacientes. La mediana de edad fue de 50 años, $51 \%$ varones. Las comorbilidades más prevalentes fueron hipertensión arterial 31,4\%, enfermedad neurológica crónica $27,1 \%$, enfermedad cardiovascular $14,4 \%$ y diabetes $13,6 \%$. Los signos y síntomas más frecuentes: fiebre $68,6 \%$ y tos $51,7 \%$. Según la severidad inicial: $33,9 \%$ neumonía moderada y $27,1 \%$ grave. El $75 \%$ de las tomografías de tórax reveló vidrio esmerilado; linfopenia presentó el 30\%. No se detectó coinfección viral. La mortalidad por todas las causas fue del $20 \%$ y del $57 \%$ en UTI con ventilación mecánica.

\begin{abstract}
${ }^{1}$ Servicio de Infectología, Sanatorio San José. Ciudad Autónoma de Buenos Aires, Argentina.

2 Dirección de Gestión de Calidad, Sanatorio San José Ciudad Autónoma de Buenos Aires, Argentina.
\end{abstract}

Autora para correspondencia: Gabriela Poblete. Email: gpoblete.infecto@gmail.com

Este trabajo no tuvo ningún tipo de financiamiento. Los contenidos del material no han sido publicados anteriormente.

Conclusiones: Nuestro trabajo describe las características y mortalidad de pacientes internados con COVID-19. Es necesario aumentar la evidencia para desarrollar modelos de predicción clínica relacionados con COVID-19.

Palabras clave: infecciones por coronavirus, pandemias, epidemiología, COVID-19. 


\section{Introducción}

El 31 de diciembre de 2019 las autoridades de la ciudad de Wuhan, en la provincia de Hubei, China, reportaron un conglomerado de 27 casos de síndrome respiratorio agudo de etiología desconocida entre personas vinculadas a un mercado de productos marinos (1). El 7 de enero de 2020 un nuevo coronavirus fue identificado, denominado SARS-CoV-2, y la enfermedad que produce, $\operatorname{COVID-19}(2,3,4)$. Tras la rápida diseminación en China y su introducción subsecuente en otros países, el virus SARS-CoV-2 se ha propagado ampliamente en la región de las Américas (5). El primer caso de COVID-19 en Argentina se confirmó el 3 de marzo, correspondiente a un viajero proveniente de Italia (6). El 11 de marzo de 2020 la Organización Mundial de la Salud declaró la pandemia por el virus SARS-CoV-2, continuando a la fecha la investigación sobre el nuevo patógeno, el espectro de manifestaciones clínicas y la gravedad de la enfermedad (7). En Argentina existe un único trabajo publicado sobre las características epidemiológicas y clínicas de pacientes con COVID-19 a nivel nacional (8). El objetivo del presente estudio es describir las características epidemiológicas, demográficas, clínicas y la mortalidad por todas las causas de los primeros casos confirmados de COVID-19 internados en el Sanatorio San José, establecimiento de salud privado, en la Ciudad Autónoma de Buenos Aires (CABA).

\section{Materiales y métodos}

Estudio retrospectivo, de corte transversal y descriptivo realizado durante el período comprendido entre el 3 de marzo, que corresponde al reporte del primer caso de COVID-19 en Argentina, y el 8 de julio de 2020, fecha de actualización del protocolo de manejo de casos sospechosos y confirmados COVID-19, versión 35, del Ministerio de Salud del Gobierno de la CABA (9). Se analizaron las características epidemiológicas, demográficas y clínicas de los registros de casos confirmados de COVID-19 notificados al SNVS 2.0 e internados en el Sanatorio San José (establecimiento de salud privado con internación de adultos, CABA) y se evaluó la mortalidad por todas las causas. Se definió caso confirmado al que había presentado RT-PCR para SARS-CoV-2 positiva en una muestra de hisopado nasofaríngeo.

Criterios de inclusión: caso confirmado de COVID-19 por RT-PCR de muestra de hisopado nasofaríngeo que cumplía criterio de caso sospechoso y se encontraba internado en el Sanatorio San José y vinculado al mismo por SNVS 2.0.

Criterios de exclusión: no cumplía criterio de caso sospechoso de COVID-19, o que continuara internado al momento del corte del estudio.

Los casos confirmados se clasificaron según antecedente epidemiológico en: importados (con antecedente de viaje en los últimos 14 días a otro país o provincia con circulación comunitaria), contacto estrecho con caso confirmado (en contacto estrecho con un caso sospechoso de COVID-19 en los últimos 14 días) o comunitarios (sin ningún viaje ni contacto estrecho con un caso en los últimos 14 días). En características demográficas se incluyeron la edad, el sexo, la ocupación (trabajador de la salud: profesional con función asistencial, técnico/auxiliar con función asistencial, o con función administrativa), la pertenencia a una residencia para personas mayores, institución de salud mental, institución penitenciaria, hospital o clínica asistencial y la residencia o no en el Área Metropolitana de Buenos Aires (AMBA).

Para la descripción de las comorbilidades se tomaron como referencia trabajos publicados en la literatura y se incluyeron en el análisis la presencia o ausencia de las siguientes: hipertensión arterial, diabetes, enfermedad cardiovascular, enfermedad respiratoria crónica, enfermedad neurológica crónica, inmunosupresión congénita o adquirida, enfermedad oncológica, insuficiencia renal crónica, obesidad, fumador o ex fumador (10-13).

Para la signo-sintomatología, clasificación de la severidad inicial y herramientas diagnósticas auxiliares se utilizaron las recomendaciones del Ministerio de Salud de la Nación (MSAL) (14).

Se consideraron los síntomas incluidos en la definición de caso sospechoso vigente en el período analizado (fiebre, tos, disnea, odinofagia, anosmia, disgeusia) (15) y se agregaron cefalea, mialgias, malestar general y síntomas gastrointestinales (diarrea y vómitos) en base a lo reportado en una revisión sistemática y metaanálisis de 148 estudios de 9 países (16).

Se clasificó la gravedad de los casos en: enfermedad no complicada (ausencia de infiltrado radiológico y de factores de riesgo, con auscultación normal y saturación de oxígeno (Sat. $\mathrm{O}_{2}$ ) >95\%); neumonía leve (edad <60 años, ausencia de comorbilidades, ausencia de inmunosupresión, compromiso radiológico unilateral, Sat. $\mathrm{O}_{2}>95 \%$, sin criterios ATS/IDSA); neumonía moderada (edad $\geq 60$ años, presencia de una o más comorbilidades, presencia de inmunosupresión, compromiso radiológico bilateral, $\mathrm{SatO}_{2} \leq 95 \%$, sin criterios ATS/IDSA); neumonía grave (presencia de criterios ATS/IDSA: uno de dos mayores (necesidad de asistencia respiratoria 
mecánica (ARM) invasiva, shock séptico (vasopresores) o tres menores (taquipnea $\geq 30 / \mathrm{min}, \mathrm{PaO}_{2} / \mathrm{FiO}_{2}<250$, confusión/ desorientación, infiltrados multilobares, urea $>42 \mathrm{mg} / \mathrm{dL}$, leucopenia $<4.000 / \mathrm{mm}^{3}$, plaquetopenia $<100.000 / \mathrm{mm}^{3}$, temperatura $<36{ }^{\circ} \mathrm{C}$, hipotensión requiriendo hidratación agresiva) (14).

La evaluación del compromiso pulmonar se realizó con radiografía de tórax (frente y perfil) y/o tomografía axial computarizada (TAC) de tórax, cuando la radiografía no permitió definir la presencia o algunas cualidades del infiltrado, y cuyo resultado hubiese tenido impacto en la toma de decisión de la conducta ulterior, de acuerdo con las recomendaciones del MSAL (14).

Para los datos de laboratorio se tuvieron en cuenta los parámetros sugeridos por las recomendaciones del MSAL que definen severidad: leucopenia $\left(<4.000 / \mathrm{mm}^{3}\right)$, linfopenia $\left(<1.000 / \mathrm{mm}^{3}\right)$, plaquetopenia $\left(<100.000 / \mathrm{mm}^{3}\right)$, elevación de urea $(>42 \mathrm{mg} / \mathrm{dL}$ ) y creatinina $(>1.1 \mathrm{mg} / \mathrm{dL}$ en mujeres $\mathrm{y}>1.2$ $\mathrm{mg} / \mathrm{dL}$ en hombres), alteración del hepatograma (bilirrubina total $>1.2 \mathrm{mg} / \mathrm{dL}$, aspartato aminotransferasa y/o alanina aminotransferasa $>40 \mathrm{U} / \mathrm{L}$ ); y la elevación de biomarcadores de severidad: ferritina $(>1.200 \mathrm{ng} / \mathrm{ml})$ y dímero $\mathrm{D}(>1.000$ $\mu \mathrm{g} / \mathrm{L})(14)$. Se incluyó el laboratorio con la mayor cantidad de parámetros considerados, realizado dentro de las primeras 48 horas desde el ingreso.

Para el tratamiento se utilizaron las recomendaciones para el abordaje terapéutico de COVID-19 del MSAL vigentes al momento del estudio. Se incluyeron: oxigenoterapia, ARM, tratamiento antiviral (lopinavir/ritonaviry/o hidroxicloroquina), corticoides, inmuno moduladores, el uso de plasma de convaleciente y el tratamiento antibiótico empírico para neumonía adquirida en la comunidad (NAC).

En todos los casos, además de la solicitud de RT-PCR para SARS-CoV-2 en la muestra de hisopado nasofaríngeo, se evaluó la existencia de coinfección viral mediante el estudio de panel viral: por inmunofluorescencia indirecta (IFI) la detección de adenovirus, y por inmunofluorescencia directa (IFD) la detección de virus sincicial respiratorio, virus influenza $B$, parainfluenza I, parainfluenza II, parainfluenza III, virus influenza $A$; y por RT-PCR la detección del virus influenza A, influenza A/H1N1 e influenza B. También se consignó la ausencia o presencia de coinfecciones bacterianas y fúngicas durante la internación.

El análisis descriptivo se realizó mediante planilla de cálculo (Microsoft ${ }^{\circledR}$ Excel ${ }^{\circledR}$ para Microsoft 365, versión 2011). Las variables continuas fueron descriptas con mediana y rango intercuartilo (RIC) y las variables categóricas con su número y porcentaje. Para el análisis estadístico se utilizó el programa STATA versión 13.0.

En cuanto a las consideraciones éticas, el estudio se realizó en adhesión a la Ley Nacional 25.326 de Protección de Datos Personales y a la Declaración de Helsinki de la Asociación Médica Mundial. Para preservar la privacidad y confidencialidad de los datos personales se generó un código para cada paciente, preservando el anonimato de todos los participantes, quienes firmaron el consentimiento informado para ser ingresados en nuestro establecimiento de acuerdo con las normativas vigentes.

\section{Resultados}

Durante el período analizado se registraron 148 casos confirmados por RT-PCR de hisopado nasofaríngeo vinculados al Sanatorio San José a través de su notificación al SNVS 2.0. De ellos, 30 pacientes fueron excluidos (25 pacientes no se internaron, 4 no cumplían criterio de caso sospechoso y 1 se encontraba internado al momento de corte del estudio). Un total de 118 pacientes fueron incluidos en el análisis.

En relación con la clasificación epidemiológica de los casos, el $66 \%$ fue de origen comunitario. La mediana de edad fue de 50 años (RIC: 33-81), con un ligero predominio del sexo masculino del $51 \%$. Del total de los casos, el $9 \%$ se reportó en trabajadores de la salud y el $25 \%$ procedía de un centro de tercer nivel (en su mayoría residencia para personas mayores). En el $100 \%$ de los casos se identificó el AMBA como lugar de residencia (Tabla 1).

\section{Tabla 1. Característica poblacional}

\begin{tabular}{|c|c|}
\hline Variables & $\begin{array}{c}\text { Todos los pacientes } \\
\qquad(n=118)\end{array}$ \\
\hline $\begin{array}{l}\text { Datos demográficos } \\
\text { Edad en años, mediana (RIC)* } \\
\text { Sexo masculino, n (\%) } \\
\text { Ocupación } \\
\text { Trabajador de la salud, n (\%) } \\
\text { Procedencia } \\
\text { Centro de tercer nivel, n (\%) } \\
\text { Residencia } \\
\text { AMBA**, n (\%) }\end{array}$ & $\begin{array}{c}50(33-81) \\
60(51) \\
11(9) \\
30(25) \\
100(100)\end{array}$ \\
\hline $\begin{array}{l}\text { Datos epidemiológicos } \\
\text { Clasificación según antecedente } \\
\text { epidemiológico } \\
\text { Comunitario, n (\%) } \\
\text { Contacto estrecho, n (\%) }\end{array}$ & $\begin{array}{l}78(66) \\
40(34)\end{array}$ \\
\hline
\end{tabular}


Respecto a la distribución de comorbilidades, en el $62 \%$ de los pacientes se registró su presencia. La más frecuente fue HTA en el $31,4 \%$ de los casos, seguida por enfermedad neurológica crónica en el $27,1 \%$, enfermedad cardiovascular en el $14,4 \%$ y diabetes en el 13,6\% (Tabla 2). En el 61\% de los pacientes se registraron dos o más comorbilidades.

Respecto a la signo-sintomatología, la más frecuente fue fiebre en el $68,6 \%$, seguido de tos en el $51,7 \%$ y anosmia en el $32 \%$ de los casos. Al evaluar la frecuencia de otros síntomas, el $31,4 \%$ presentó malestar general, el $27,1 \%$ odinofagia, el $22 \%$ disnea, el $21,2 \%$ cefalea, el $21,2 \%$ mialgias, el $16,1 \%$ disgeusia y el 6,8\% síntomas gastrointestinales (Tabla 3).

Al clasificar los casos según la severidad inicial de acuerdo con las recomendaciones del MSAL, el 27,1\% correspondió a enfermedad no complicada, el $18,6 \%$ a neumonía leve, el $33,9 \%$ a neumonía moderada y el $27,1 \%$ a neumonía grave.

Se realizó radiografía de tórax a todos los pacientes, y en el 52\% se complementó la evaluación del compromiso pulmonar con TAC de tórax. En el 17\% de los casos se encontró neumonía unilateral y en el $38 \%$ neumonía bilateral. Al analizar los hallazgos en la TAC de tórax se informó la presencia de vidrio esmerilado en el $75 \%$ de los estudios.

Respecto a los hallazgos de laboratorio, el $8 \%$ de los pacientes presentó leucopenia, el $30 \%$ linfopenia y plaquetopenia se observó en el 3\% de los casos. En el 31\% de los pacientes

\section{Tabla 2. Comorbilidades}

\begin{tabular}{|c|c|}
\hline Comorbilidad, n (\%) & $\begin{array}{l}\text { Todos los pacientes } \\
\qquad(n=118)\end{array}$ \\
\hline Alguna & $71(62)$ \\
\hline $\mathrm{HTA}^{*}$ & $37(31,4)$ \\
\hline Enfermedad neurológica crónica & $32(27,1)$ \\
\hline Enfermedad cardiovascular & $17(14,4)$ \\
\hline $\mathrm{DBT}^{\star \star *}$ & $16(13,6)$ \\
\hline Inmunosupresión & $14(11,9)$ \\
\hline Ex fumador & $10(8,5)$ \\
\hline Enfermedad oncológica & $6(5,1)$ \\
\hline Fumador & $5(4,2)$ \\
\hline 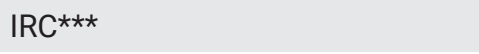 & $5(4,2)$ \\
\hline Enfermedad respiratoria crónica & $4(3,4)$ \\
\hline Obesidad & $0(0)$ \\
\hline
\end{tabular}

\begin{tabular}{|c|c|}
\hline Variable, n (\%) & $\begin{array}{c}\text { Todos los pacientes } \\
\qquad(\mathrm{n}=118)\end{array}$ \\
\hline Fiebre & $81(68,6)$ \\
\hline Tos & $61(51,7)$ \\
\hline Anosmia & $38(32)$ \\
\hline Malestar general & $37(31,4)$ \\
\hline Odinofagia & $32(27,1)$ \\
\hline Disnea & $26(22)$ \\
\hline Cefalea & $25(21,2)$ \\
\hline Mialgias & $25(21,2)$ \\
\hline Disgeusia & $19(16,1)$ \\
\hline Síntomas gastrointestinales & $8(6,8)$ \\
\hline
\end{tabular}

se detectó elevación de urea, y en el $16 \%$ de creatinina. En 115 casos se pudo evaluar el hepatograma y se registró su alteración en el $6 \%$ a expensas del aumento de la bilirrubina total y en el $17 \%$ por elevación de las transaminasas. Dentro de los biomarcadores de severidad se solicitó ferritina en 53 pacientes y se registró que el $13 \%$ tenía un valor mayor a 1.200 $\mathrm{ng} / \mathrm{ml}$, en tanto, el dímero $D$ fue solicitado en 27 pacientes y el $48 \%$ tenía un valor mayor a $1.000 \mu / L$.

Alconsiderarelabordajeterapéutico delos pacientes internados por COVID-19, en el 5\% de los casos se indicó tratamiento antiviral (lopinavir/ritonavir con o sin hidroxicloroquina), el 4\% de los pacientes recibió plasma de convaleciente y al $9 \%$ se le administró corticoides. Tocilizumab e ivermectina fueron indicados en un paciente cada uno. El $30 \%$ de los pacientes requirió oxigenoterapia y en el $58 \%$ se indicó tratamiento antibiótico empírico para NAC.

En ninguno de los casos analizados se detectó la presencia de coinfección viral por influenza u otros virus respiratorios. Y durante la internación, un $14 \%$ de los pacientes presentaron coinfección bacteriana y/o fúngica (15 pacientes bacteriana y dos pacientes fúngica, siendo una de estas bacteriana y fúngica).

La mediana de días de internación global fue de 7.5 (RIC: 4-15). Un total de 19 pacientes requirieron internación en unidad de terapia intensiva (UTI), y siete de ellos necesitaron ARM. Entre los pacientes incluidos en el análisis, la mortalidad por todas las causas fue del $20 \%(n=24)$. Del total de muertes, el $71 \%$ ocurrieron en pacientes de 80 años y más. Al evaluar la mortalidad de los internados en UTI vinculados a ARM se registró una mortalidad del $57 \%$. 


\section{Discusión}

La enfermedad por el virus SARS-CoV-2, COVID-19, se ha convertido en un problema emergente para la salud mundial. En Argentina, los datos publicados en pacientes adultos internados son escasos.

Comparado con el estudio nacional de mayores dimensiones (116.974 casos) publicado (8), la mediana de edad encontrada de 50 años fue mayor que la reportada de 37 años, pero con similitud en la distribución por sexo, siendo el 51\% varones.

En concordancia con el estudio de Rearte y col. (8) y las experiencias locales de Pulido y col. (17) y Castro y col. (18), la comorbilidad más frecuente fue HTA. En nuestra serie, la enfermedad neurológica crónica ocupó el segundo lugar en frecuencia $(27,1 \%)$, siendo mayor que lo reportado en estudios previos (13).

Entre los signos y síntomas, los más relevantes fueron fiebre, tos y anosmia. En el $68,6 \%$ de los pacientes se reportó fiebre, encontrándose similitud con los trabajos nacionales $(8,17,18)$ y la literatura internacional (16).

En referencia a las herramientas auxiliares diagnósticas, en el 75\% de los pacientes en quienes se realizó TAC de tórax se informó vidrio esmerilado y el 30\% presentó linfopenia, hallazgos similares fueron encontrados en estudios realizados en China $(19,20)$.

En nuestra serie no se documentó la presencia de coinfección viral que fue pesquisada activamente al ingreso de todos los pacientes. En contexto de la pandemia de COVID-19, a nivel nacional más del $99 \%$ de las muestras positivas analizadas a la fecha corresponde a SARS-CoV-2, permaneciendo baja la detección de virus influenza y demás virus respiratorios (21).

La mortalidad por todas las causas fue del $20 \%$, similar a la reportada en otros estudios observacionales del 21-26\% (22-25). La mortalidad en los pacientes internados en UTI vinculados a ARM fue del 57,1\%, mayor que la reportada en la experiencia de González y col. (25) en la provincia de Buenos Aires, del $44,6 \%$. En dicho trabajo la mortalidad de pacientes en UTI vinculados a ARM y con shock séptico fue del 55,2\%, y esta categorización no fue realizada en nuestro estudio. Considerando la edad promedio de la población estudiada, podríamos mencionar que la inmunosenescencia jugaría un rol independiente en la evolución de estos pacientes. carácter retrospectivo, hubo datos ausentes al analizar las historias clínicas, en particular del laboratorio. La descripción de los cuadros graves es limitada por el bajo número de pacientes en dicho grupo, y al haber sido realizado en un único centro, podría verse limitado el alcance de nuestros hallazgos. Sin embargo, los resultados son concordantes con lo descrito previamente en la literatura.

Nuestro objetivo es continuar de manera prospectiva para alcanzar mayor robustez metodológica y proponer el desarrollo de modelos de predicción clínica para el diagnóstico y pronóstico de pacientes con COVID-19.

\section{Reconocimientos}

Los autores agradecemos a todo el personal del Sanatorio San José por el esfuerzo y dedicación en su desempeño laboral.

Dentro de las limitaciones del presente estudio, dado el 


\section{Bibliografía}

1. Zhu N, Zhang D, Wang W, et al. A Novel Coronavirus from Patients with Pneumonia in China, 2019. N Engl J Med 2020; 382: 727-33.

2. Wu $\mathrm{Y}, \mathrm{Ho}$ W, Huang $\mathrm{Y}$, et al. SARS-CoV-2 is an appropriate name for the new coronavirus. Lancet 2020; 395: 949-50.

3. Wang C, Horby PW, Hayden FG, Gao GF. A novel coronavirus outbreak of global health concern. Lancet 2020; 395: 470-73.

4. Li Q, Guan X, Wu P, et al. Early Transmission Dynamics in Wuhan, China, of Novel Coronavirus-Infected Pneumonia. N Engl J Med 2020; 382: 1199-1207.

5. Ministerio de Salud Argentina. Alerta Epidemiológico 22 de enero de 2020 SE04. https://www.argentina.gob.ar/sites/ default/files/20200123-coronavirus-alerta-epidemiologicaargentina.pdf; consultado agosto 2020.

6. Ministerio de Salud. Salud confirma el primer caso de coronavirus en el país. En: https://www.argentina.gob.ar/ noticias/salud-confirma-el-primer-caso-de-coronarivus-en-elpaís; consultado agosto 2020.

7. WHO Director-General opening remarks at the media briefing on COVID-19- 11 March 2020. En: https://www.who. int/es/ dg/speeches/detail/who-director-general-s-openingremarksat-the-media-briefing-on-covid-19-11-march2020; consultado agosto 2020.

8. Rearte A, Baldani AEM, Barcena Barbeira $P$, et al. Carracterísticas epidemiológicas de los primeros 116974 casos de COVID-19 en Argentina, 2020. Rev Argent Salud Publica 2020; 12 Supl COVID-19:e5.

9. Ministerio de Salud del Gobierno de la Ciudad Autónoma de Buenos Aires. Protocolo de manejo de casos sospechosos y confirmados COVID-19. (Versión 35. 08/07/2020).

10. Huang $C$, Wang $Y$, Li $X$, et al. Clinical features of patients infected with 2019 novel coronavirus in Wuhan, China. Lancet. 2020; 395: 497-506.

11. Chen N, Zhou M, Dong X, et al. Epidemiological and clinical characteristics of 99 cases of 2019 novel coronavirus pneumonia in Wuhan, China: a descriptive study. Lancet 2020; 395: 507-513.

12. Zhonghua Liu Xing Bing Xue Za Zhi. Epidemiology Working Group for NCIP Epidemic Response, Chinese Center for Disease Control and Prevention. [The epidemiological characteristics of an outbreak of 2019 novel coronavirus diseases (COVID-19) in China]. 2020 Feb 10;41(2):145-151. Chinese.

13. Rodriguez-Morales AJ, Cardona-Ospina JA, GutiérrezOcampo E, et al. Clinical, laboratory and imaging features of COVID-19: a systematic review and meta-analysis. Travel Med Infect Dis 2020; 34: 101623.

14. Ministerio de Salud de la Nación. Recomendaciones para equipos de salud. Nuevo Coronavirus 2019-nCoV. Buenos Aires: MSAL; 2020. [Disponible en: https://www. argentina.gob.ar/salud/recomendaciones-equipos-de-saludcoronavirus-2019-ncov]

15. Ministerio de Salud de la Nación. Recomendaciones para equipos de salud. Definición de caso [Internet]. Buenos Aires: MSAL; 2020 [actualizado 6 Jul 2020]. Disponible en: https://www.argentina.gob.ar/salud/coronavirus-COVID-19/ definicion-de-caso

16. Grant MC, Geoghegan L, Arbyn M, et al. The Prevalence of Symptoms in 24,410 Adults Infected by the Novel Coro־navirus (SARS-CoV-2; COVID-19): a systematic review and meta-analysis of 148 studies from 9 countries. PLoS One 2020; 15: e0234765.

17. Pulido L, Solís-Aramayo MA, Ibarrola M, et al. Experiencia inicial en la atención de pacientes con COVID-19 en un Hospital privado de alta complejidad de la Ciudad de Buenos Aires. Medicina (B Aires) 2020; 80: 433-8.

18. Castro $H$, Canale $H$, Ferreyro $B$, et al. Características clínicas de la enfermedad por coronavirus 2019 en un centro de Argentina. Cohorte retrospectiva. Medicina(B Aires) 2020;80.

19. Kui L, Fang YY, Deng Y, et al. Clinical characteristics of novel coronavirus cases in tertiary hospitals in Hubei Province. Chin Med J (Engl). 2020.

20. Shi $\mathrm{H}$, et al. Radiological findings from 81 patients with COVID-19 pneumonia in Wuhan, China: a descriptive study. The Lancet Infectious Diseases (2020).

21. Boletín Integrado de Vigilancia. Edición semanal. $N^{\circ} 514$ SE 38/2020. Ministerio de Salud de la Nación. Disponible en: https://www.argentina.gob.ar/salud/epidemiologia/ boletines2020

22. Casas-RojoJM,Antón-SantosJM,Millán-Núñez-CortésJ,etal. Clinical characteristics of patients hospitalized with COVID-19 in Spain: results from the SEMI-COVID-19 Registry. Rev Clin Esp (Barc). 2020 Sep 9. doi: 10.1016/j.rceng.2020.07.003. Epub ahead of print. PMCID: PMC7480740.

23. Fried MW, Crawford JM, Mospan AR, et al. Patient Characteristics and Outcomes of 11,721 Patients with COVID19 Hospitalized Across the United States. Clin Infect Dis. 2020 Aug 28:ciaa1268.

24. Docherty AB, Harrison EM, Green CA, et al. Features of 20133 UK patients in hospital with covid-19 using the ISARIC WHO Clinical Characterisation Protocol: prospective observational cohort study. BMJ. 2020; 369:m1985.

25. González SE, Regairaz L, Ferrando NS, et al. Terapia con plasma de convalecientes en pacientes COVID-19 en la Provincia de Buenos Aires. Medicina (B Aires). 2020; 80(5): 417-424. 
Epidemiological and clinical characteristics of patients hospitalized with COVID-19 in Buenos Aires city

Introduction: The COVID-19 pandemic represents a global health challenge and continues to be investigated.

Objective: To describe the epidemiological, demographic, clinical characteristics and all-cause mortality of patients hospitalized with COVID-19, in a private health care facility in Buenos Aires city.

Materials and methods: A retrospective, cross-sectional, and descriptive study was conducted between March 3 and July 8 , 2020. Adult patients with a confirmed diagnosis of COVID-19 by nasopharyngeal swab RT-PCR, admitted to the general ward and intensive care unit (ICU) at the San José Sanatorium were included. Epidemiological, demographic (age, sex, occupation, origin, residence), clinical characteristics, and all-cause mortality were analyzed. Continuous variables were described with median and interquartile range (IQR) and categorical variables with number and percentage. The STATA v 13.0 program was used.

Results: 118 patients were included. The median age was 50 years, $51 \%$ were men. The most prevalent comorbidities: arterial hypertension $31.4 \%$, chronic neurological disease $27.1 \%$, cardiovascular disease $14.4 \%$ and diabetes $13.6 \%$. The most frequent signs and symptoms: fever $68.6 \%$ and cough $51.7 \%$. According to the initial severity: $33.9 \%$ moderate pneumonia and $27.1 \%$ severe. Ground glass was reported in $75 \%$ of chest scans; lymphopenia presented $30 \%$. Viral coinfection was not detected. Mortality from all causes was $20 \%$, and $57 \%$ in ICU with mechanical ventilation.

Conclusions: Our work describes the characteristics and mortality of hospitalized patients with COVID-19. Increased evidence is needed to develop clinical predictive models related to COVID-19.

Keywords: coronavirus infections, pandemics, epidemiology, COVID-19. 